\title{
Simulating the Realization of a Mixed Clock Ensemble
}

\author{
Christian Trainotti, Tobias D. Schmidt, and Johann Furthner \\ Institute of Communications and Navigation, \\ German Aerospace Center (DLR), \\ Oberpfaffenhofen, Germany \\ christian.trainotti@dlr.de
}

\begin{abstract}
Global navigation satellite systems need a stable and robust system time in order to provide services with high accuracy. A well established approach for the generation of such a time scale is the composite clock method. Differently from single clock approaches, a set of clocks is used to define a system time scale by generating a weighted average of their single contributions. The clocks are measured with respect to each other and the measured signals are fed into a Kalman filter, which implicitly provides the system time of the ensemble, in terms of the implicit ensemble mean (IEM), a so called paper clock. The IEM generally exhibits a better stability than every single clock in the ensemble for all sample intervals. However, this quantity is not directly available in hardware, but can be realized by steering a clock with a dedicated control loop containing a second Kalman filter and a regulator. The output of the steered clock provides in this way a physical realization of the IEM.
\end{abstract}

The system realizing the IEM is quite complex and the resulting performances depend on a variety of factors. Furthermore, the resulting behavior can only be evaluated after a sufficient amount of data is collected, which can take a long time. For these reasons, it is desirable to have a tool for simulating this system and thus analyzing the resulting IEM before launching extensive measurements. This paper describes how such a system can be simulated, by providing a flexible environment to prove a given setup of clocks and settings.

The simulation algorithm is tested with scenarios of increasing complexity: firstly, for validation purposes, the simulation results are compared with real measurements. Secondly, different compositions of mixed ensembles are simulated. Then, the simulation is run by using different regulators and different parameters. Finally, the effects of mismodelling on the resulting IEM and its realization are assessed.

In light of the long term goal of setting up a mixed clock ensemble in hardware, these analyses can reduce the invested time, as well as pointing out which aspects of the composition algorithm must be researched with particular care.

Index Terms-Timing, clocks, time measurement, simulation, satellite navigation systems.

\section{INTRODUCTION}

Many applications today need a stable and precise time scale to ensure correct operation, such as navigation systems, finance and information systems. The composite clock technique based on Kalman filters, proposed by Brown [1], is a promising concept to achieve these requirements. Instead of relying on a single master clock which supplies the system time, this technique considers a set of clocks, all contributing to the generation of a time scale. The clocks are measured with respect to each other and the measurements are fed into a Kalman filter which estimates the clocks' states. The filter also provides a weighted average of the contribution of each clock, called Implicit Ensemble Mean (IEM). The IEM generally outperforms each clock in the ensemble in terms of overlapping Allan deviation (OADEV) and it shows an enhanced robustness, since a failure in a clock can be compensated by the remaining clocks [2], 3]. However, the IEM is only a paper clock, in fact it computation requires knowing the unobservable phase state of each clock. It can be computed in a simulation, but in a real case, in order to have a physical realization, a clock signal has to be steered to the IEM by a dedicated control loop [2]. A second Kalman filter is needed to provide the input to a regulator, so that a clock signal is steered to the IEM and thus can be used as a physical realization for the system time.

The aim of this work is to simulate the generation and realization of the IEM of a mixed ensemble, that is, a set of clocks of different types. In the simulation it is possible to test different scenarios, for example involving different ensembles, different steering techniques and steering parameters. This reduces the need of running extensive and time consuming measurement campaigns. Furthermore, the simulation allows to test and study new techniques and algorithms, as well as mitigate unwanted effects, such as the additional noise introduced by the steering action.

This paper is structured as follows. In Section II the system under study is described in detail. Section III describes the general process of the simulation, which can be divided in blocks. Each block is introduced and their 
detailed description is contained in the following sections: Section IV] deals with the generation of the clocks' models, Section $\bar{V}$ lists the steps followed in the main simulation process, while Section VI describes how the results are evaluated. Section $\mathrm{V}$ is further divided, so that each subsection describes one block of the system: the generation of the measurements (Section $\mathrm{V}-\mathrm{A}$, the first Kalman filter (Section $\mathrm{V-B}$ ), and the second Kalman filter with the regulator (Section V-C). The performances of the simulation algorithm are tested in Section VII the validation is described in Section VII-A the realization of mixed ensembles in Section VII-B the realization using different regulators in Section VII-C and the effects of mismodelling in Section VII-D. Finally, Section VIII concludes this work and describes its outlook.

\section{Description of the System}

The system can be roughly divided in two parts: the first part consists of the clock ensemble and its estimation, while the second is responsible for IEM realization and contains the feedback loop. The scheme of the system can be seen in Fig. 1. The black solid part describes how the clock ensemble is estimated. A set of $N$ clocks of different types are measured with respect to the first clock, leading to a set of $N_{Z}=N-1$ measurements. These are fed into a Kalman filter, which estimates the states of each clock based on the stochastic clock models. The red dashed part is responsible for the realization of the IEM, as proposed in [2] The signal coming from a clock of the ensemble (or an external additional clock) is splitted and then steered by a microphase stepper. The steered signal is the physical realization of the IEM. The feedback loop consists of a second Kalman filter which estimates the states of the steered clock, and the estimation is used by the regulator to compute the control action.

\section{Simulation Setup}

The simulation algorithm in MATLAB allows a flexible environment in which the realization setup can be tested and validated. It is composed of the following steps:

1) Definition of the parameters: in the beginning the user has to set the values of all the parameters which define the simulated scenario. In particular, the ensemble must be formed (number and type of clocks), the measurement noise, the steering parameters and the length of the simulation must be set;

2) Generation of the models: with the parameters previously defined, in this step the matrices of the models and the noise vectors are generated. Three clock models are used: the 'generation model' defines how the real clocks and their measurements are simulated, the 'estimation model' is used in the first Kalman filter to estimate the clock states and the 'steering model' is used in the second Kalman filter in the feedback loop. Having different models for generating the measurements and estimating the clock states reflects the real case, where the clock model in the Kalman filter is always affected by uncertainty with respect to the real behavior of the clocks;

3) Allocation of the variables: pre-allocating the memory space for the resulting variables greatly reduces the simulation time. The size of the variables can be computed depending on the simulation parameters;

4) Execution of the simulated process: here the composition algorithm is run step by step in a loop simulating a discrete time operation. In particular, at each time step, the actions described in Section $\mathrm{V}$ are executed;

5) Post-processing and data visualization: the data collected in the simulation loop are processed to generate meaningful descriptions of the performance. In particular, plots of the OADEV of various signals are generated.

The following sections will describe these steps with more details.

\section{Definition of the Parameters and Genera- TION OF THE MODELS}

In the beginning, the user defines all the desired parameters for the simulation. In particular, the ensemble composition is set by listing the types and order of the clocks. The first clock is considered to be the reference clock, to which all the other clocks are measured, while the last one is the steered clock, whose signal corresponds to the IEM realization and it is not part of the ensemble.

The clock ensemble is modelled with a stochastic linear dynamic model, namely the 2 -state model with constant frequency drift, as described in [4]. In particular, at the time step $t_{k}$, the states of the clock $i$ at the next time step $t_{k}+\tau_{0}$ can be computed as

$$
\boldsymbol{x}_{i}\left(t_{k}+\tau_{0}\right)=\boldsymbol{\Phi}_{i} \boldsymbol{x}_{i}\left(t_{k}\right)+\boldsymbol{D}_{i}+\boldsymbol{B}_{i} u\left(t_{k}\right)+\boldsymbol{w}_{i}\left(t_{k}\right),
$$

where $\tau_{0}$ is the time discretization, $\boldsymbol{x}_{i}$ is the state vector (containing the phase deviation, part of frequency deviation and possibly a set of Markov processes), $\boldsymbol{\Phi}_{i}$ the state propagation matrix, $\boldsymbol{D}_{i}$ the drift vector, $\boldsymbol{B}_{i}$ the input matrix, $u$ the steering action and $\boldsymbol{w}_{i}$ the noise vector. The latter is normally distributed $\boldsymbol{w}_{i} \sim \mathcal{N}\left(0, \boldsymbol{Q}_{i}\left(\tau_{0}\right)\right)$ :

$$
\boldsymbol{Q}_{i}\left(\tau_{0}\right)=\left[\begin{array}{cc}
{ }_{i} \sigma_{1}^{2} \tau_{0}+{ }_{i} \sigma_{2}^{2} \frac{\tau_{0}^{2}}{3} & { }_{i} \sigma_{2}^{2} \frac{\tau_{0}^{2}}{2} \\
{ }_{i} \sigma_{2}^{2} \frac{\tau_{0}^{2}}{2} & { }_{i} \sigma_{2}^{2} \tau_{0}
\end{array}\right],
$$

where the matrix can be possibly expanded to account for a set of Markov processes. In (2), ${ }_{i} \sigma_{1}$ and ${ }_{i} \sigma_{2}$ are the noise parameters defining the clock model, together with the drift ${ }_{i} d$ and the parameters of the Markov processes ${ }_{i} U,{ }_{i} R$. The set of parameters is $\left\{{ }_{i} \sigma_{1},{ }_{i} \sigma_{2},{ }_{i} d,\left[{ }_{i} U_{1},{ }_{i} R_{1},\right], \ldots,\left[{ }_{i} U_{M},{ }_{i} R_{M},\right]\right\}$, where a set of $M$ Markov processes is considered. For more details, the 


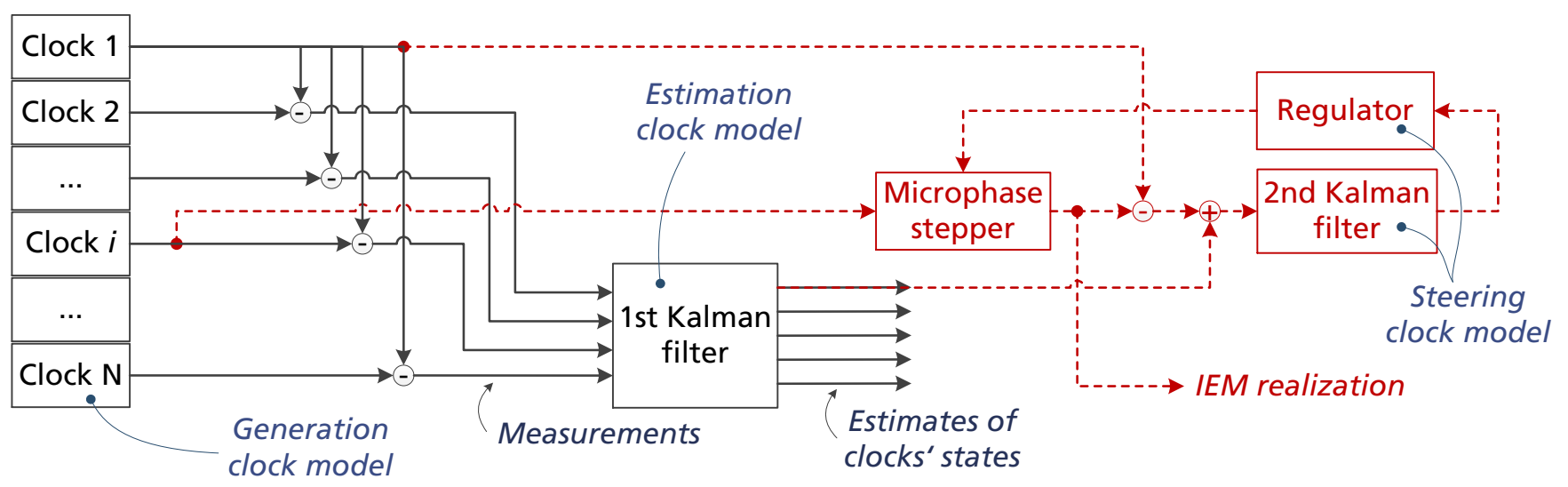

Figure 1: Scheme of the IEM realizazion.

reader can refer to [4], 5]. The clock ensemble is generated by staking the state vectors of the single clocks

$$
\boldsymbol{x}^{T}=\left[\boldsymbol{x}_{1}^{T}, \ldots, \boldsymbol{x}_{i}^{T}, \ldots, \boldsymbol{x}_{N}^{T}\right],
$$

so that the overall state equation can be formed:

$$
\boldsymbol{x}\left(t_{k}+\tau_{0}\right)=\boldsymbol{\Phi} \boldsymbol{x}\left(t_{k}\right)+\boldsymbol{D}+\boldsymbol{B} u\left(t_{k}\right)+\boldsymbol{w}\left(t_{k}\right),
$$

where the matrices and vector are computed by appropriately using the respective terms of the single clocks:

$$
\begin{gathered}
\boldsymbol{\Phi}=\operatorname{diag}\left(\boldsymbol{\Phi}_{1}, \ldots, \boldsymbol{\Phi}_{N}\right), \quad \boldsymbol{D}^{T}=\left[\boldsymbol{D}_{1}^{T}, \ldots, \boldsymbol{D}_{N}^{T}\right], \\
\boldsymbol{w} \sim \mathcal{N}\left(0, \boldsymbol{Q}\left(\tau_{0}\right)\right), \quad \boldsymbol{B}^{T}=\left[\boldsymbol{B}_{1}^{T}, \ldots, \boldsymbol{B}_{N}^{T}\right], \\
\boldsymbol{Q}\left(\tau_{0}\right)=\operatorname{diag}\left(\boldsymbol{Q}_{1}\left(\tau_{0}\right), \ldots, \boldsymbol{Q}_{N}\left(\tau_{0}\right)\right) .
\end{gathered}
$$

The clock measurement device is modelled with the measurement equation

$$
\boldsymbol{z}\left(t_{k}\right)=\boldsymbol{H} \boldsymbol{x}\left(t_{k}\right)+\boldsymbol{v}\left(t_{k}\right),
$$

where $\boldsymbol{z}$ is a vector of size $1 \times N_{Z}$ containing the measurements, $\boldsymbol{H}$ the measurement matrix and $\boldsymbol{v}$ the measurement noise. The noise has distribution $\boldsymbol{v} \sim\left(0, R_{Z} \boldsymbol{I}_{N_{Z}}\right)$, where $R_{Z}$ is the noise covariance and $\boldsymbol{I}_{i}$ indicates an identity matrix of size $i$. The measurement matrix describe how the clocks are measured: in this case the first clock will be used as a reference, to which all the other signal are measured. Each measurement consists of the difference between the phase components of each clock and the first clock. This models the current setup of the hardware measurement unit, which uses the first channel as reference, to which all others are measured. By changing the $\boldsymbol{H}$ matrix, other setups can be modelled. If no Markov process is considered, then

$$
\boldsymbol{H}=\left[\begin{array}{ccccccccc}
-1 & 0 & 1 & 0 & 0 & 0 & \ldots & 0 & 0 \\
-1 & 0 & 0 & 0 & 1 & 0 & \ldots & 0 & 0 \\
\vdots & & & & & & & & \\
-1 & 0 & 0 & 0 & 0 & 0 & \ldots & 1 & 0
\end{array}\right]
$$

The actual number of zeros on each row depends on the number of Markov processes that are used to model the different clocks. The gain matrix of the regulator $\boldsymbol{K}$ can be computed by using control theory techniques, once the model of the steered clock is defined. In this work, the approaches of pole placement (PP) and linear-quadratic Gaussian regulator (LQG) are considered. As described in [6], [7], [8], these are two promising techniques for clock steering, which have been already extensively tested. Their usage in clock steering is described in 7], where a similar analysis is conducted, but for the case of a clock being steered to another one. Other works on the topic are for example [9], [10], [11], [12], 13], 14]. In the case of PP, the user can set the desired position of the closed-loop poles by choosing the parameter $0<\lambda<1$. For the LQG method, the weights $W_{R}$ and $\boldsymbol{W}_{Q}$ must be set. $W_{R}$ is a scalar weight for the control value, while $\boldsymbol{W}_{Q}$ is a matrix of weights for each of the states of the steered clock. Both methods yield the gain matrix $\boldsymbol{K}$, which is multiplied by the estimated states of the steered clock $\hat{\boldsymbol{x}}_{N}\left(t_{k}\right)$ produced by the second Kalman filter to obtain the control action:

$$
u\left(t_{k}\right)=-\boldsymbol{K} \cdot \hat{\boldsymbol{x}}_{N}\left(t_{k}\right) .
$$

The set of matrices and parameters which completely describe the system is defined as

$$
\mathcal{M}_{j}=\left\{\boldsymbol{\Phi}_{j}, \boldsymbol{D}_{j}, \boldsymbol{B}_{j}, \boldsymbol{Q}_{j}, \boldsymbol{H}_{j},{ }_{j} R_{Z}\right\}
$$

As already mentioned, three models of the clock ensemble are generated: the model $\mathcal{M}_{g}$ is used to generate the measurements, $\mathcal{M}_{e}$ is used in the first Kalman filter, while $\mathcal{M}_{s}$ is used in the second Kalman filter and for the regulator's design.

\section{Simulation of the Process}

The main part of the simulation consists of a loop which is executed at every time step. Within the loop, all the 


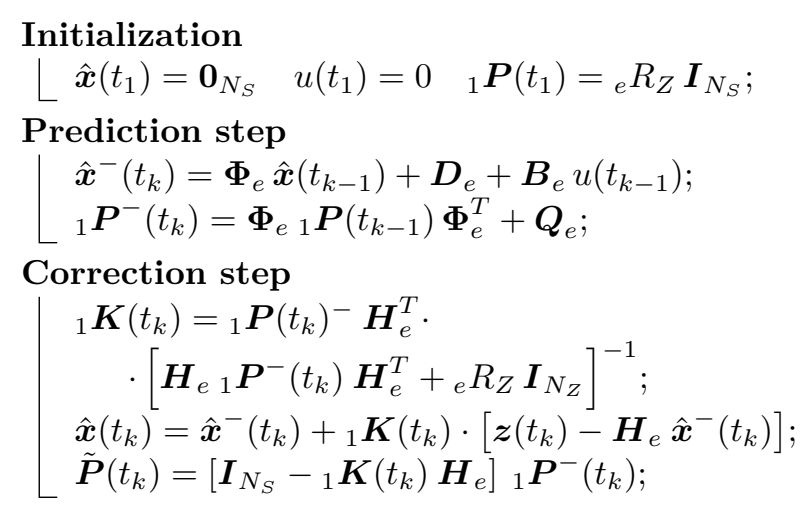

\section{IEM generation}

$$
\begin{aligned}
& \boldsymbol{a}\left(t_{k}\right)=\frac{\boldsymbol{P}_{\varphi \varphi}^{-1}\left(t_{k}\right) \mathbf{1}}{\mathbf{1}^{T} \boldsymbol{P}_{\varphi \varphi}^{-1}\left(t_{k}\right) \mathbf{1}} ; \\
& \operatorname{IEM}\left(t_{k}\right)=\boldsymbol{a}\left(t_{k}\right) \cdot\left[\boldsymbol{\varphi}\left(t_{k}\right)-\hat{\boldsymbol{\varphi}}\left(t_{k}\right)\right] ;
\end{aligned}
$$

\section{Covariance reduction}

$$
\left\lfloor\begin{array}{l}
\boldsymbol{P}_{R}=\boldsymbol{S} \tilde{\boldsymbol{P}}\left(t_{k}\right) \boldsymbol{S}^{T} \\
{ }_{1} \boldsymbol{P}\left(t_{k}\right)=\frac{1}{2}\left(\boldsymbol{P}_{R}+\boldsymbol{P}_{R}^{T}\right)
\end{array}\right.
$$

Initialization
$\left\lfloor\hat{\boldsymbol{\xi}}\left(t_{1}\right)=\mathbf{0}_{N_{S}} ; \tilde{u}\left(t_{1}\right)=0 ;{ }_{2} \boldsymbol{P}\left(t_{1}\right)={ }_{s} R_{Z} \boldsymbol{I}_{N_{S}} ; \ell=1 ;\right.$
if $\left(t_{k} \bmod T_{u}==0\right)$ then

$$
\begin{aligned}
& \text { Prediction step } \\
& \begin{array}{l}
\hat{\boldsymbol{\xi}}^{-}\left(t_{\ell}\right)=\boldsymbol{\Phi}_{s} \hat{\boldsymbol{\xi}}\left(t_{\ell-1}\right)+\boldsymbol{D}_{s}+\boldsymbol{B}_{s} \tilde{u}\left(t_{\ell-1}\right) ; \\
{ }_{2} \boldsymbol{P}\left(t_{\ell}\right)^{-}=\boldsymbol{\Phi}_{s 2} \boldsymbol{P}\left(t_{\ell-1}\right) \boldsymbol{\Phi}_{s}^{T}+\boldsymbol{Q}_{s} ;
\end{array} \\
& \text { Correction step } \\
& \begin{array}{l}
{ }_{2} \boldsymbol{K}\left(t_{\ell}\right)={ }_{2} \boldsymbol{P}\left(t_{\ell}\right)^{-} \boldsymbol{H}_{s}^{T} . \\
\quad\left[\boldsymbol{H}_{s 2} \boldsymbol{P}\left(t_{\ell}\right)^{-} \boldsymbol{H}_{s}^{T}+{ }_{s} R_{Z} \boldsymbol{I}_{N_{Z}}\right]^{-1} ; \\
\hat{\boldsymbol{\xi}}\left(t_{\ell}\right)=\hat{\boldsymbol{\xi}}^{-}\left(t_{\ell}\right)+{ }_{2} \boldsymbol{K}\left(t_{\ell}\right) \cdot\left[\tilde{\boldsymbol{z}}\left(t_{k}\right)-\boldsymbol{H}_{s} \hat{\boldsymbol{\xi}}^{-}\left(t_{\ell}\right)\right] ; \\
\tilde{\boldsymbol{P}}\left(t_{\ell}\right)=\left[\boldsymbol{I}_{N_{S}}-{ }_{2} \boldsymbol{K}\left(t_{\ell}\right) \boldsymbol{H}_{s}\right] \cdot{ }_{2} \boldsymbol{P}\left(t_{\ell}\right)^{-} ;
\end{array}
\end{aligned}
$$

\section{Correction step}

Covariance reduction

$$
\begin{aligned}
& \boldsymbol{P}_{R}=\boldsymbol{S} \tilde{\boldsymbol{P}}\left(t_{\ell}\right) \boldsymbol{S}^{T} ; \\
& { }_{2} \boldsymbol{P}\left(t_{\ell}\right)=\frac{1}{2}\left(\boldsymbol{P}_{R}+\boldsymbol{P}_{R}^{T}\right) ;
\end{aligned}
$$

\section{Compute steering}

$$
\begin{aligned}
& \left\lfloor\begin{array}{l}
u\left(t_{k}\right)=\tilde{u}\left(t_{\ell}\right)=-\boldsymbol{K} \hat{\boldsymbol{\xi}}_{S}^{-}\left(t_{\ell}\right) ; \\
\ell=\ell+1 ;
\end{array}\right. \\
& \text { else } \\
& \left\lfloor u\left(t_{k}\right)=0 ;\right.
\end{aligned}
$$

(a) First Kalman filter

(b) Second Kalman filter

Figure 2: Steps in the two Kalman filters

blocks shown in Figure 1 are processed. In particular, the simulation follows these steps:

1) the clocks and their measurements evolve according to the generation and steering models;

2) the clocks' states are estimated by the first Kalman filter based on the generated measurements and on the estimation model. Additionally, in this Kalman filter the IEM is computed. This value only serves for postprocessing purposes and not for steering, since it can not be recreated in real hardware;

3) the measurement of the steered clock is modified to achieve the realization of IEM;

4) the states of the steered clock are estimated by the second Kalman filter based on the modified measurement and on the steering model;

5) the control action for next step is computed.

These steps are described in more details in the following subsections.

\section{A. Generation of the Measurements}

The simulated states of the clocks and their measurements are generated by following the (3) and the (4), using the generation model $\mathcal{M}_{g}$. These quantities are considered as being the real states and measurements:

$$
\begin{aligned}
& \boldsymbol{x}_{k}=\boldsymbol{\Phi}_{g} \boldsymbol{x}_{k-1}+\boldsymbol{D}_{g}+\boldsymbol{B}_{g} u_{k-1}+{ }_{g} \boldsymbol{w}_{k-1 s}, \\
& \boldsymbol{z}_{k}=\boldsymbol{H}_{g} \boldsymbol{x}_{k}+{ }_{g} \boldsymbol{v}_{k},
\end{aligned}
$$

where the noise vectors ${ }_{g} \boldsymbol{w}$ and ${ }_{g} \boldsymbol{v}$ are normally distributed:

$$
{ }_{g} \boldsymbol{w}_{k} \sim \mathcal{N}\left(\boldsymbol{Q}_{g}\right), \quad{ }_{g} \boldsymbol{v}_{k} \sim \mathcal{N}\left({ }_{g} R_{Z} \boldsymbol{I}_{N_{Z}}\right) .
$$

\section{B. First Kalman Filter}

The first Kalman filter estimates the states of the clocks in the ensemble based on the estimation model $\mathcal{M}_{e}$ and on the measurements. It can either use generated measurements or real measurements, obtained from the corresponding hardware ensemble being simulated. Two steps in the Kalman filter are of great importance: firstly, the IEM has to be generated using the real and estimated states and the covariance matrix. Secondly, the covariance matrix must be reduced, since the clock ensemble is an unobservable system, as described in [5]. Please note that the step for IEM generation is possible only when using generated states and measurements, since in hardware only phase and frequency differences are available. Let $\varphi$ be a vector containing the phase component of each clock from the states vector, and $\boldsymbol{P}_{\varphi \varphi}$ a matrix containing only the elements corresponding to the phase component of each clock from the covariance matrix $\tilde{\boldsymbol{P}}_{k}$. Then, the first Kalman filter follows the steps depicted in the algorithm of Fig. 2a where $\boldsymbol{S}$ is a diagonal matrix deleting the phase components in the covariance matrix $\tilde{\boldsymbol{P}}[5], \mathbf{1}$ is a column vector of ones, and $N_{S}$ the number of states in $\boldsymbol{x}$. The hat $\hat{\boldsymbol{\bullet}}$ notation indicates the a posteriori estimation of a quantity, 
while the minus $\bullet^{-}$indicates the a priori estimation. The subscripts aim to distinguish quantities of the first and second filter and different clock models.

\section{Second Kalman Filter and Regulator}

The second Kalman filter estimates the states of the clocks by considering a modified measurement of the steered clock. In particular, following [3], the new measurement is defined as:

$$
\tilde{\boldsymbol{z}}_{k}=\left[\begin{array}{llll}
z_{k, 1} & \ldots & z_{k, N_{Z}-1} & z_{k, N_{Z}}+\hat{x}_{k, 1}
\end{array}\right]^{T} .
$$

In other words, the last component of the measurement vector (corresponding to the steered clock) is summed with the estimated first state, that is the estimated phase of the reference clock. The modified measurement is used to estimate a new set of states in the second Kalman filter. The second Kalman filter computes the control action, instead of the IEM. It is not executed at each step, but only when the control action has to be applied, that is every $T_{u}$ steps. This happens at the step $k$ if $k$ is a multiple of the control interval $T_{u}$. The steps of the filter are listed in Fig. $2 \mathrm{~b}$ where $\hat{\boldsymbol{\xi}}_{S}^{-}\left(t_{\ell}\right)$ is the last part of the vector $\hat{\boldsymbol{\xi}}^{-}\left(t_{\ell}\right)$, with the states of the steered clock. Please note that the prediction step does not use the last value of $u$, but that of $\tilde{u}$. While the vector $u$ contains zeros at the time steps where the control is not computed, $\tilde{u}$ contains only the values computed in the second Kalman filter $(\tilde{u} \subseteq u)$. The control value is stored and applied at the next step, so that the feedback loop is closed.

\section{Postprocessing}

During each step of the simulation, the variables are saved for their successive analysis. The most important metric used here is the OADEV [15], that is computed as function of the sampling interval $\tau$ and shown in log-log plot. In these logarithmic plots it is possible to directly identify the characteristic noise components of each signal [4], 15], 16], 17]. In the following, these stabilities are computed and plotted:

- the OADEV of the clocks $\sigma_{i}(\tau)$, computed using the simulated phase $\varphi_{i}$, or the OADEV of the real measurements;

- the theoretical OADEV $\sigma_{i}^{g}(\tau)$ of the model used to generate the measurement $\left(\mathcal{M}_{g}\right)$;

- the theoretical OADEV $\sigma_{i}^{e}(\tau)$ of the model used to estimate the clock states $\left(\mathcal{M}_{e}\right)$.

The theoretical OADEVs are computed starting from the model parameters [4]:

$$
\begin{aligned}
\sigma_{i}(\tau)= & {\left[\frac{{ }_{i} \sigma_{1}^{2}}{\tau}+\frac{{ }_{i} \sigma_{2}^{2}}{3} \tau+\frac{\tau^{2}}{2}{ }_{i} d+\sum_{j=1}^{M} \frac{{ }_{i} U_{j}}{R^{2} \tau^{2}}[-3\right.} \\
& \left.\left.+4 \exp \left(-{ }_{i} R_{j} \tau\right)-\exp \left(-2{ }_{i} R_{j} \tau\right)+2_{i} R_{j} \tau\right]\right]^{\frac{1}{2}} .
\end{aligned}
$$

\section{Testing of the Simulation Algorithm}

In this section, the simulation algorithm is validated and tested. For validation purposes, a set of real measurement from an IEM realization of an homogeneous ensemble is used. The same setup is simulated and the results are compared in terms of OADEV. Then, the algorithm is tested with scenarios of increasing complexity: in the beginning, various mixed ensemble are simulated; after that, various sets of parameters are tested on a given ensemble, in order to evaluate the performances in the different cases: firstly, the parameters of the regulator are changed, using either PP or LQG with different coefficients. Secondly, the effects of clock mismodelling can be assessed by generating the measurements and estimating the clock states using two different models.

\section{A. Validation with an Homogeneous Ensemble}

In order to validate the simulation algorithm, a real measurement of an ensemble of 3 rubidium clocks (Spectratime SRO-100) is compared with the results of the corresponding simulation. A validation involving a mixed ensemble can not be performed yet, since the hardware realization of a mixed clock ensemble is still being implemented in our time lab. However, in this study an Oven Controlled Quartz Oscillator (OCXO, KL3381 by $\mathrm{K}+\mathrm{K}$ Messtechnik GmbH) is steered via a High Resolution Offset Generator (SpectraDynamics HROG-10) to the IEM of 3 rubidium clocks. In the hardware Kalman filter, the clocks are modelled with a 2-state clock model without drift or Markov processes (Rubidium: $\sigma_{1}^{2}=1.53 \cdot 10^{-23}, \sigma_{2}^{2}=2.8 \cdot 10^{-27}$. OCXO: $\left.\sigma_{1}^{2}=10^{-24}, \sigma_{2}^{2}=5 \cdot 10^{-26}\right)$. The regulator is a $\mathrm{LQG}$ with parameters $W_{R}=1, \boldsymbol{W}_{Q}=\operatorname{diag}\left(10^{-20}, 1\right)$, applying a control action every 10 seconds. The modelled measurement noise covariance is $R=10^{-24}$. This setup was run for 72 hours and the resulting stability is shown in Fig. 3a. Two measurement devices are in use: the first is referenced to an Active Hydrogen Maser (AHM, by KVARZ-VREMYA) so that the stability of the single clocks can be assessed; the second device is referenced to the first rubidium, so that the measurements used in the IEM generation can be collected. These can be used in the simulation as described in the following.

The behavior of the OCXO is very abnormal, since it does not present any drift at long term. The rubidium instead show an increased drifting behavior. Nevertheless, the system succeeds in steering the OCXO to the expected IEM behavior. The steered OCXO is characterized by a relatively high short term stability and a bump due to the steering process. Its long term behavior resembles more an average of the rubidium clocks, rather than an IEM with improved stability. However, this latter behavior can be seen also in the simulation in Fig. $3 \mathrm{~b}$. The simulation is setup to resemble the real case as much as possible. The estimation model corresponds to the one used in the hardware realization (2-state model without Markov 


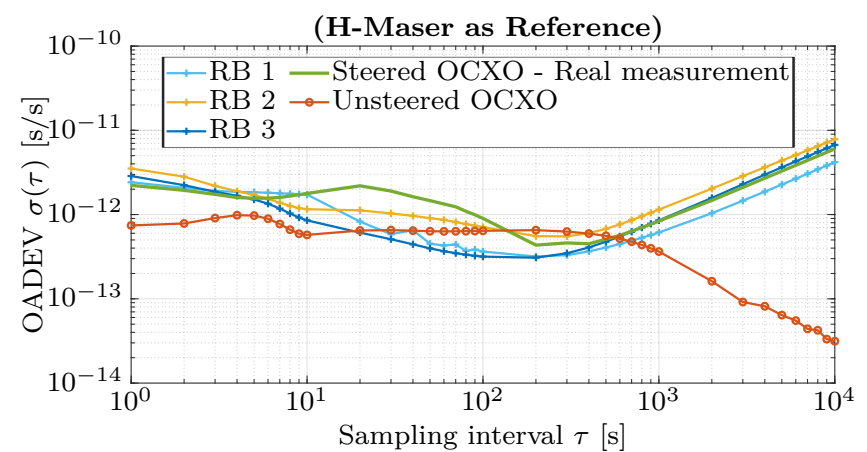

(a) Measured realization: using real measurements and real steering.

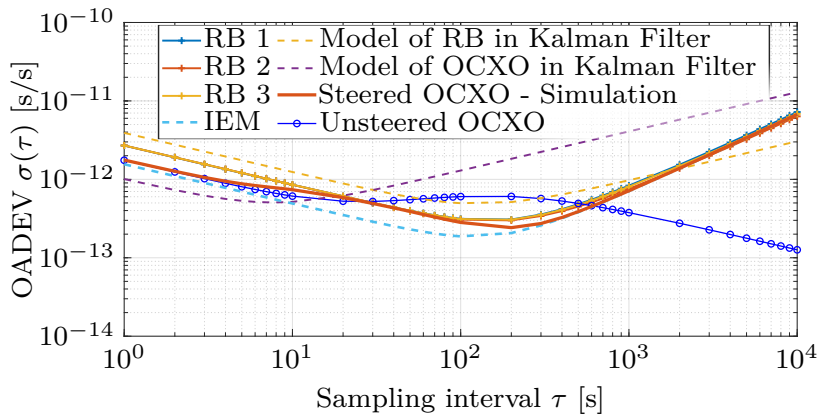

(b) Simulated realization: using simulated measurements and simulated steering.

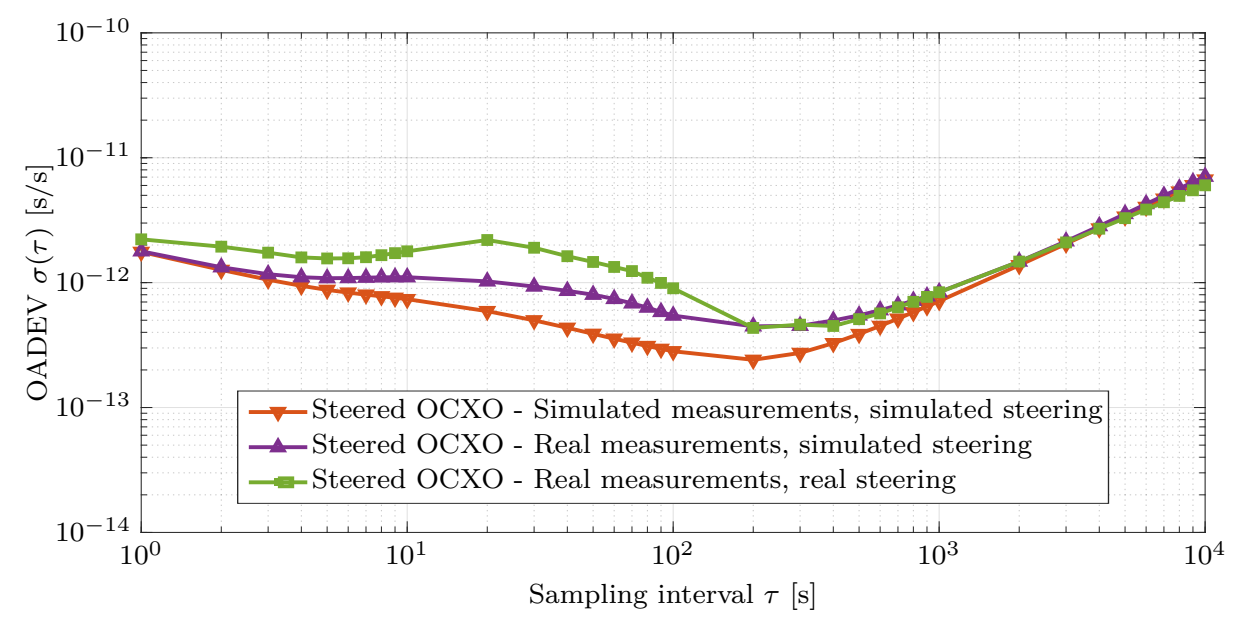

(c) Comparison of the realizations: real, simulated and simulated with real measurements.

Figure 3: Validation of the algorithm by comparing the real measurement and the simulation of the realization of an ensemble of 3 rubidium clocks.

processes or drift), while the generation model was found by fitting the measurements [4, producing the behaviors shown in solid marked lines. The regulator is designed using LQG with the aforementioned parameters, and the simulated measurements have a noise with covariance $R_{Z}=$ $R=10^{-24}$. The simulated IEM (and therefore also the steered clock) shows a behavior at long term which does not improve the stability of the rubidium clocks. This could be due to the enhanced drift. It however shows a good agreement with the real case. A difference with the real case is the generated measurement noise; in the simulation the generated noise has the same covariance as in the estimation model $\left(R_{Z}=R\right)$, which could be different in hardware.

A similar simulation is performed by including the real measurements: the simulated clocks, Kalman filters and regulator are setup in the same way, but instead of using the simulated (generated) measurements to estimate the clocks' states in the first Kalman filter, the real measurements are introduced. In this way it is not possible to compute the IEM, since the real states of the clocks are not available.
The resulting stability of the simulated steered clock can be seen in Fig. $3 \mathrm{c}$ the short term stability is the same as the purely simulated case (since the steered clocks are the same in both cases), but considering the real measurements increases the magnitude of the steering bump. This could imply that the steering process in hardware introduces some additional effects which are not regarded in the simulation and requires further analyses. Nevertheless, the three cases show a good agreement in terms of OADEV of the steered clock, and an almost perfect overlapping at long terms. The successful comparison of the real measurements and the corresponding simulation serves as an initial validation of the simulation algorithm.

\section{B. Realization of the IEM of Mixed Ensembles}

Various scenarios of mixed ensembles have been simulated. The ensembles are generated by combining clocks showing good short term stability together with clocks having good long term stability. This choice produces an IEM profiting from both clocks and possibly having good stability for a long set of sampling intervals. The clock with 


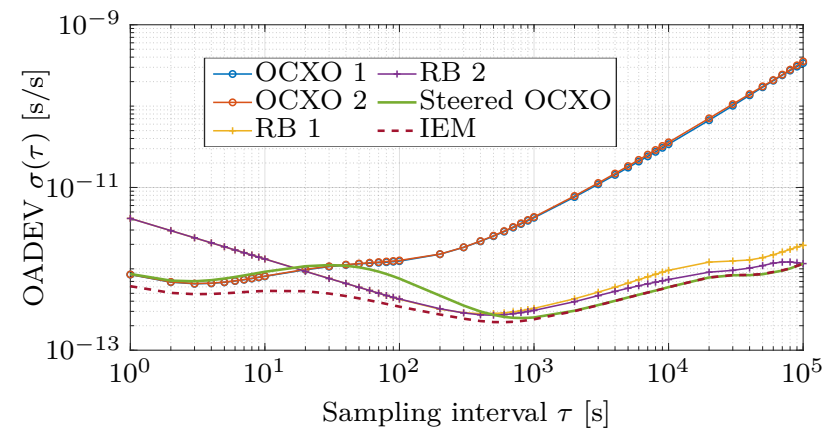

(a) An OCXO realizes an ensemble of 2 OCXOs and 2 rubidium clocks.

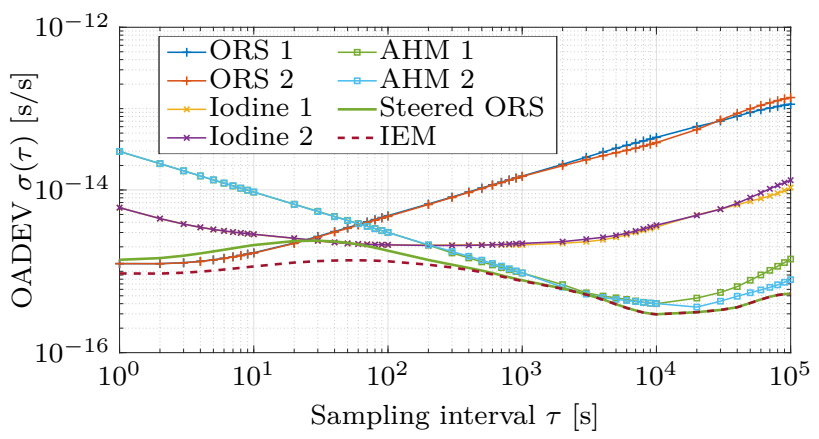

(c) An ORS realizes an ensemble of 2 ORS, 2 iodine clocks and 2 AHMs.

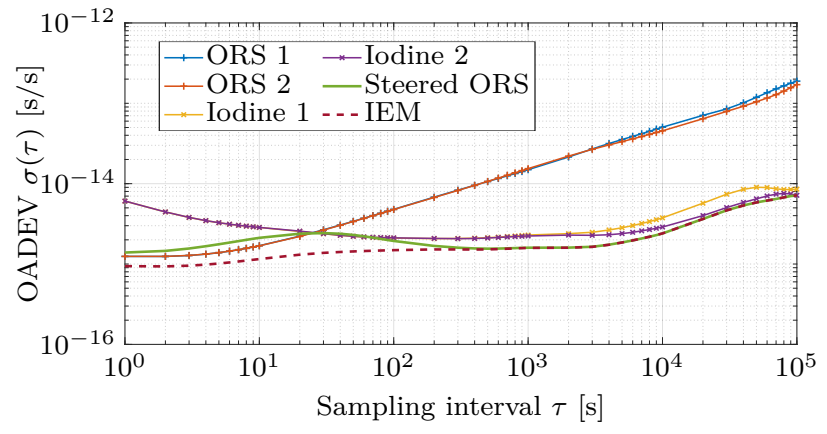

(b) An ORS realizes an ensemble of 2 ORS and 2 iodine clocks.

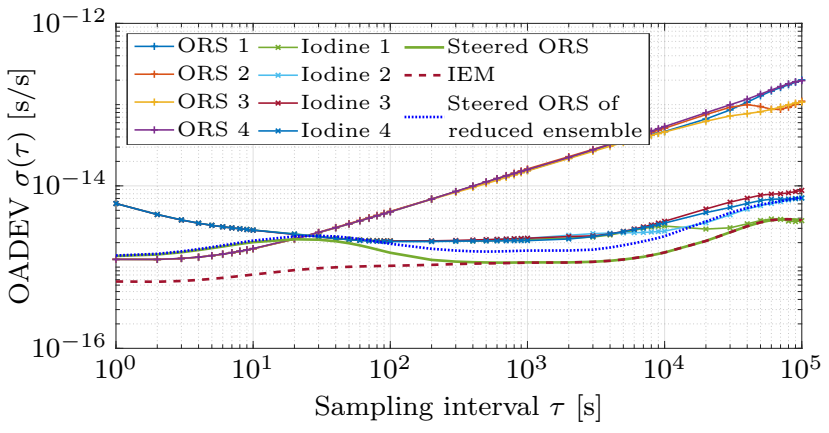

(d) An ORS realizes an ensemble of 4 ORs and 4 iodine clocks. The steered ORS of the reduced ensemble corresponds to the case in Fig. $4 b$

Figure 4: Different examples of mixed IEM realizations. The regulator is designed with pole placement $(\mathrm{PP}), \lambda=0.6$, control interval $T_{u}=10 \mathrm{~s}$. In each plot, the dashed line represents the corresponding IEM, while the solid line without markers is the steered clock.

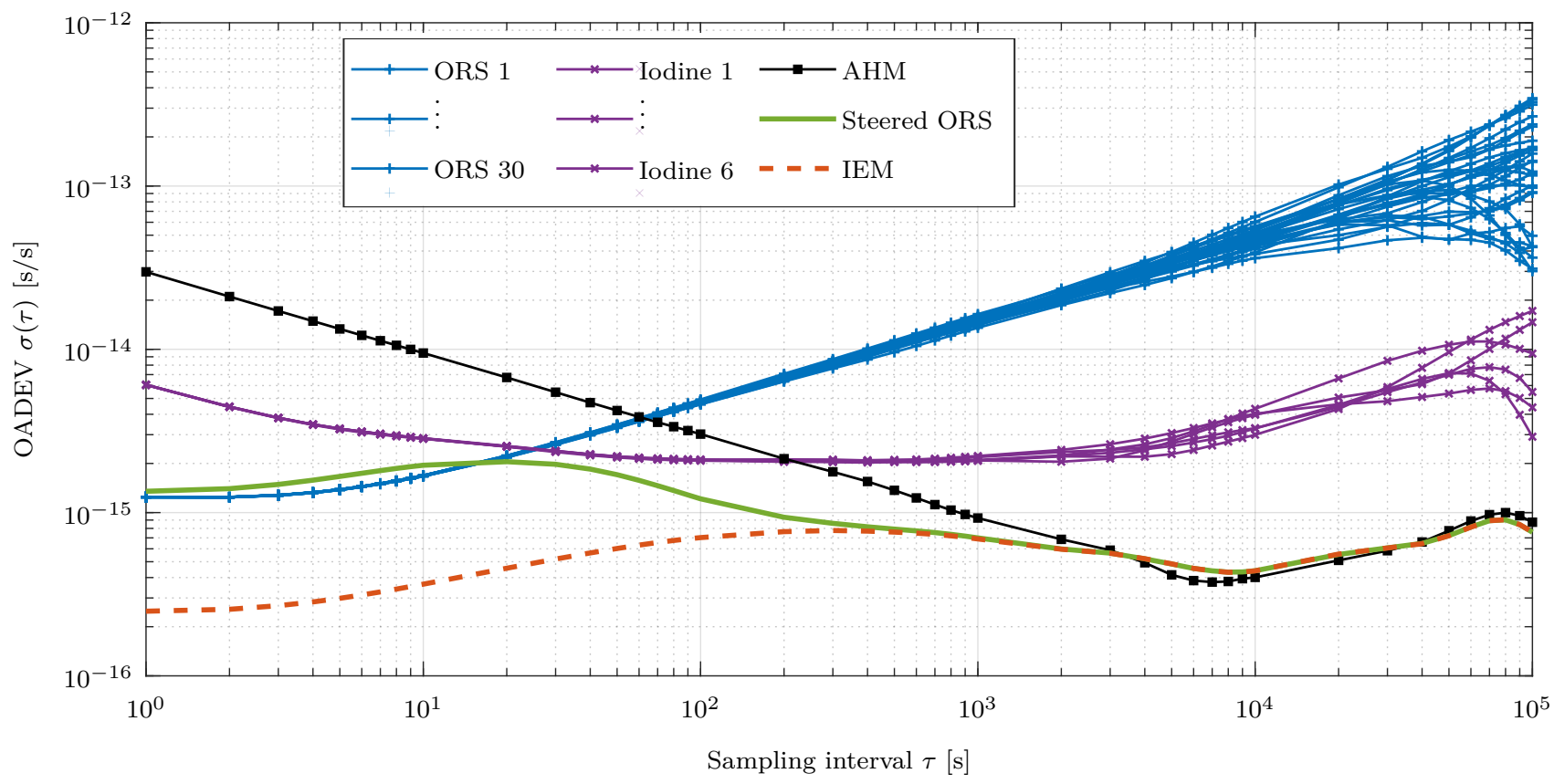

Figure 5: Realization of Kepler system time: ensemble of 30 cavity-stabilized laser systems (ORS), 6 iodine clocks and 1 Active Hydrogen Maser (AHM). 
the best short term stability in each ensemble is chosen as steered clock; the reason for this can be understood by considering the first example in Fig. 4a The ensemble of 2 OCXOs and 2 rubidium clocks has an IEM shown with dashed line. The steered OCXO (solid green line) reaches the IEM at a sample interval of about $10^{3} \mathrm{~s}$. However, at short sample times, the realization has a worse stability than the IEM. This has two reasons: first, the steered clock at short terms has the same stability of the original unsteered signal, since the control feedback can not improve the short term stability, being applied every $10 \mathrm{sec}-$ onds. Secondly, the steering process introduces some jumps which, albeit small, worsen the stability of the steered signal producing a bump at sampling intervals around the control interval 22. These two effects can be seen in every simulation of this paper, as well as in the measurements. If the goal is to have a steered signal with improved stability, it is therefore desirable to steer clocks showing a good short term stability and choosing a control interval having the smallest possible impact on the resulting OADEV.

Figure 4 shows 4 scenarios with different mixed ensembles: in Fig. 4a an OCXO realizes an ensemble of 2 OCXOs and 2 rubidium clocks; in Fig. $4 \mathrm{~b}$ a cavitystabilized laser system (Optical Reference System - ORS) realizes an ensemble of 2 ORSs and 2 optical clocks based on molecular iodine (iodine clocks); in Fig. 4c an ORS realizes an ensemble of 2 ORSs, 2 iodine clocks and 2 AHMs; Fig. 4d shows an extension of the second case, where an ORS realizes an ensemble of 4 ORSs and 4 iodine clocks. In all cases, the clock with the best short term stability is steered and the regulators are designed using PP with $\lambda=0.6$ and a control interval of $10 \mathrm{~s}$. The case in Fig. $4 \mathrm{~d}$ is an extension of the case in Fig. 4b, where the number of clocks of each type is increased from 2 to 4 . The realization of the ensemble of 4 clocks is shown with the dotted blue line, while the solid green line is for the ensemble of 8 clocks. It can be noted that increasing the number of clocks produces an IEM with better stability, but the short term stability of the steered clock is not improved, due to the aforementioned reasons.

An additional case has been simulated, which involves a larger number of clocks. The aim is to reproduce a new concept for a future GNSS, called Kepler [18], [19], 20], and to assess which stability can be achieved in such a scenario. In the Kepler system, 24 navigation satellites carry an ORS each. The system performances benefit from 6 additional satellites flying in low earth orbit. They carry the same ORS in addition to optical clocks based on molecular iodine. Additionally, an AHM is located at one ground station. All the satellites are connected via inter-satellite links (ISL), so that it is possible to tightly synchronize them to a common system time. Kepler system time (KST) can be conceived of being the IEM of all the clocks in the system, profiting from the contribution of in total 37 clocks. Each satellite produces its local copy of KST, by steering an ORS signal towards the IEM. This is possible since the satellite receives the information about the frequency references on the other satellites thanks to the ISLs. In this way, the KST is produced onboard each satellite, which is synchronized to it. The Kepler clock ensemble and its realization is simulated using $\mathrm{PP}\left(\lambda=0.6\right.$ and $\left.T_{u}=10 \mathrm{~s}\right)$ and the resulting stability is shown in Fig. 5. It can be noted that the IEM shows a stability in terms of OADEV smaller than $10^{-15} \mathrm{~s} / \mathrm{s}$ for all computed sampling intervals. However, the realization has a slightly worse stability than the IEM at short terms due to the effects of the steering process. At long terms the IEM shows a behavior which lies above the stability of the AHM. This is probably because the difference between the clocks' stabilities is quite big (two orders of magnitude) and the contribution of the AHM to the IEM is smaller compared to the combined weight of 30 ORS.

\section{Realization Using Different Designs of the Regulator}

In this section, the effect of using different control parameters is investigated. The ensemble composition is kept constant and consists of an OCXO which is steered to the IEM of 2 OCXOs and 2 rubidium clocks. Fig. 6 shows the effects of changing the control interval $T_{u}$ on the steered clock. In the case of PP (Fig. 6a) the $\lambda$ parameter is set to $\lambda=0.6$. For LQG (Fig. 6b) the weights are:

$$
W_{R}=10^{-8}, W_{Q}=\left(\begin{array}{ccc}
10^{-20} & & 0 \\
& 1 & \\
0 & & 10^{-35}
\end{array}\right) \text {. }
$$

As expected, changing the control interval causes a shift along the $\tau$ axis of the bump in the stability curve of the realization. The PP method appears to be more sensitive to different control intervals, while the LQG method shows more consistent results. Please note that the differences among the curves at long terms are due to the statistical nature of the simulations.

In Fig. 7, various realizations using different parameters for regulator design are shown. For PP, the parameter $\lambda$ was gradually increased from $\lambda=0.2$ to $\lambda=0.8$. As can be seen in Fig. 7a, when increasing $\lambda$ the steering bump moves to longer sampling intervals. For LQG, the analysis is more complex, since there are more parameters coming into play and there exist only rules of thumb for choosing them. In Fig. 7b] a simplified case is considered, where the Markov processes are neglected. In this way, there are 3 parameters to be set: the weight $W_{R}$ and the weights in the matrix $\boldsymbol{W}_{Q}$. In total, 18 cases have been simulated by combining different values of the weights. The resulting realizations showing similar behaviors are collected together (shown in the simplified legend with curly brackets). In the first set of simulations, the weight of the second state is set to $\boldsymbol{W}_{Q}(2,2)=1$ and the other weights are changed: this doesn't influence the realizations, which are overlapping each other (black solid lines). The red lines show the second 


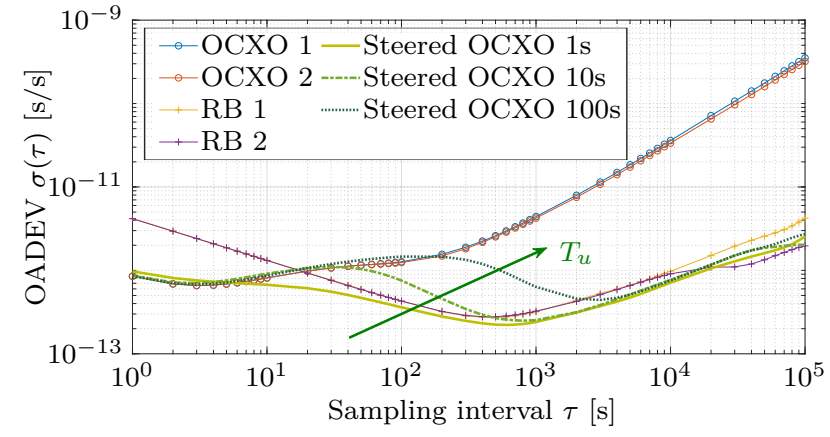

(a) Steering using PP, $\lambda=0.6$.

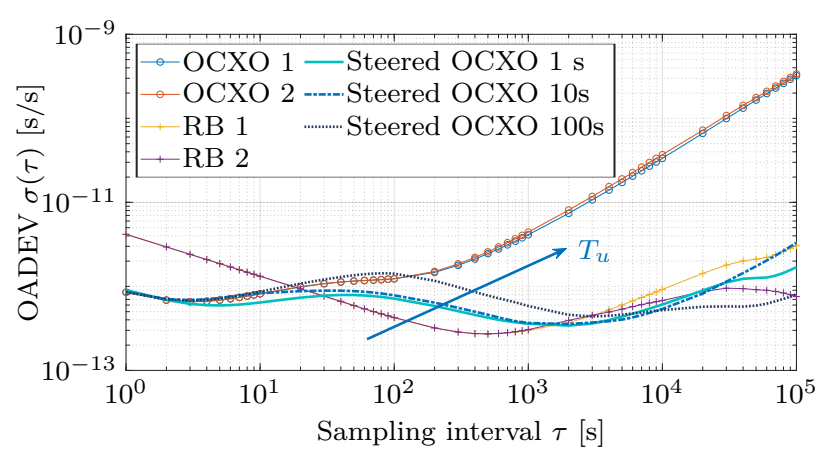

(b) Steering using LQG, parameters of the [5].

Figure 6: Effects on the IEM realization by using increasing control intervals: $T_{u}=\{1 \mathrm{~s}, 10 \mathrm{~s}, 100 \mathrm{~s}\}$

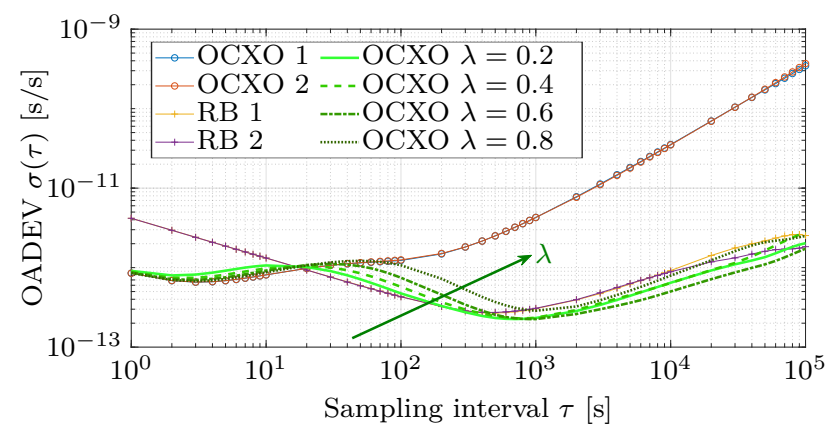

(a) Steering using PP with different $\lambda$.

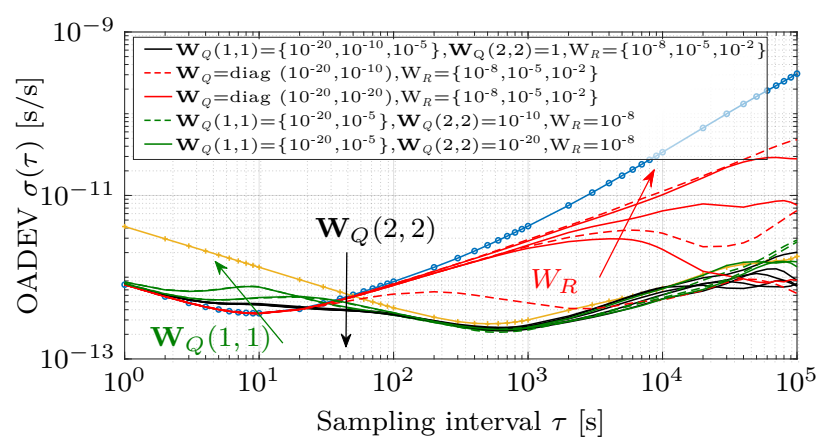

(b) Steering using LQG with different weights.

Figure 7: Effects on the IEM realization by using different parameters. In case of PP, an increasing $\lambda=\{0.2,0.4,0.6,0.8\}$. In case of LQG, refer to the legend. $T_{u}=10 \mathrm{~s}$.

set of simulations, where $W_{R}$ is changed and $\boldsymbol{W}_{Q}$ set to 2 different values: $\boldsymbol{W}_{Q}(2,2)=10^{-10}$ (dashed lines) and $\boldsymbol{W}_{Q}(2,2)=10^{-20}$ (solid lines). In both cases, by increasing $W_{R}$ the stability of the steered clock will be similar to the unsteered one, since the steering action is reduced. The realization shows therefore an increase at long terms, tending to the unsteered OCXO. The change in $W_{R}$ is more impacting when $\boldsymbol{W}_{Q}(2,2)$ is higher. Finally, the green lines show the cases with constant $W_{R}$. Again, the dashed lines are for $\boldsymbol{W}_{Q}(2,2)=10^{-10}$ and the solid ones for $\boldsymbol{W}_{Q}(2,2)=10^{-20}$. In this case, the dashed and solid green lines are overlapping when the same value of $\boldsymbol{W}_{Q}(1,1)$ is used, suggesting that they are not influenced by $\boldsymbol{W}_{Q}(2,2)$. An increase of the first weight generates a shift in the steering bump towards shorter sampling intervals.

\section{Effects of Mismodelling}

The Kalman filters and the generation of the measurements are performed using two separate models in order to reproduce a real case scenario, where it is impossible to perfectly predict the clock behavior and the model used in the Kalman filter is approximated. In order to evaluate the effect of this mismodelling, the simulation has been run with an intentionally incorrect estimation model. The first analysis concerns the clock models: in one case, the estimation model has a worse stability than the one used to generate the measurements. Every parameter in the model (except the Markov parameters ${ }_{i} R_{j}$, setting the processes' locations along the $\tau$-axis) has been increased by 10 times. The models' stabilities are shown in Fig. $8 \mathrm{a}$ using dashed marked lines. It can be noted that the IEM (and consequently the realization) is sensible to an increase of the drift parameter in the estimation model. In the other case, the estimation model has a better stability than the generation model. Every parameter in the model (except ${ }_{i} R_{j}$ ) has been decreased by 100 times. The mismodelling does not significantly change the realization, as shown in Fig. $8 \mathrm{~b}$

The second analysis focuses on the variance of the measurement noise. It appears in the Kalman filter with the parameter $R$, and in the measurement generation with the parameter $R_{Z}$. Keeping the latter constant, the value in the estimation model is increased and decreased by 3 or 6 orders of magnitude starting from $R_{z}$. The resulting realization is compared with the case of a perfect model $\left(R=R_{Z}\right)$ : in Fig. $8 \mathrm{c} R_{z}$ is set to $10^{-21}$, while in Fig. $8 \mathrm{~d}$ it is set to $10^{-27}$. It can be noted that if the noise $R$ is modelled with a lower parameter than the actual one $\left(R \ll R_{Z}\right)$, the IEM and the corresponding realization are not particularly 

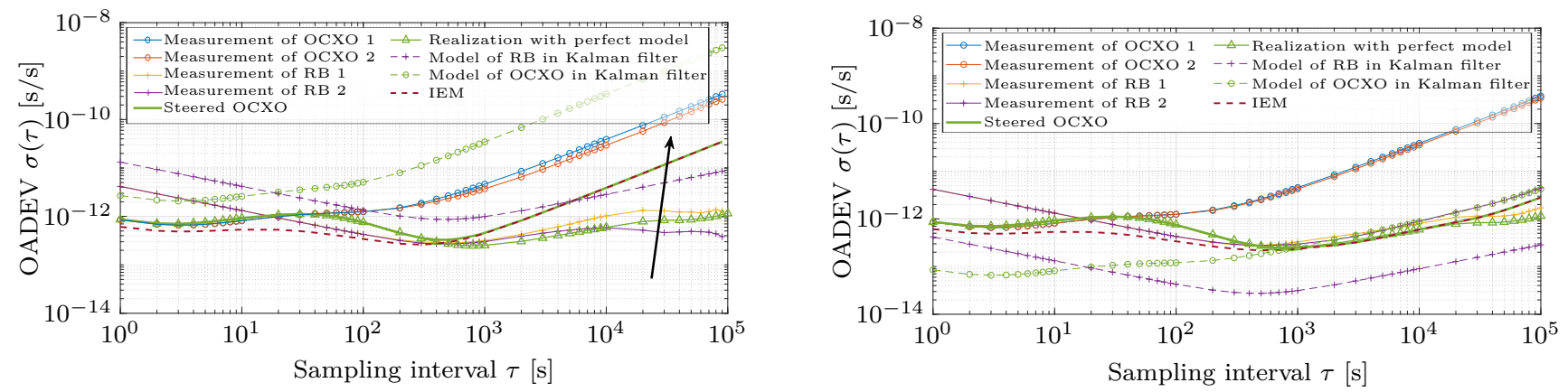

(a) Clock model in the $\mathrm{KF} \mathcal{M}_{e}$ has worse stability than the model (b) Clock model in the KF $\mathcal{M}_{e}$ has better stability than the model $\mathcal{M}_{g}$ used to generate the clocks.

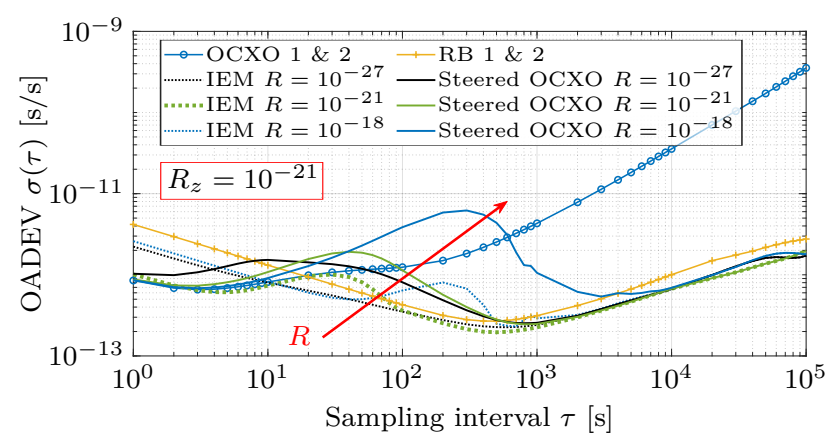
$\mathcal{M}_{g}$ used to generate the clocks.

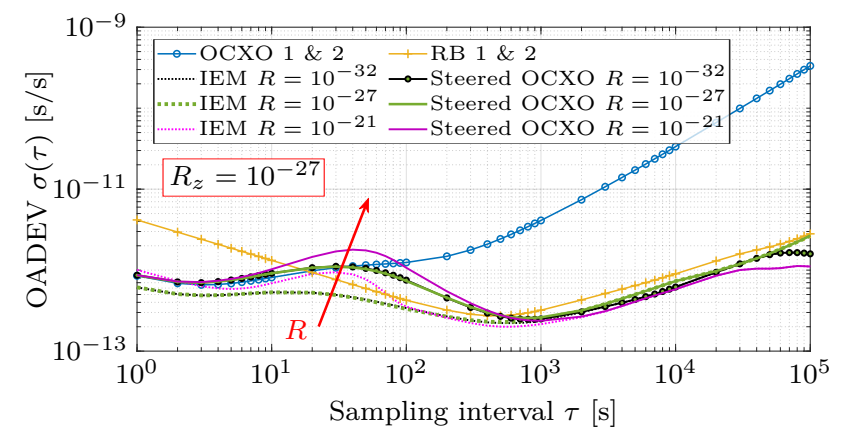

(c) Mismodelling of the measurement noise $R$, with $R_{Z}=10^{-21}$.

(d) Mismodelling of the measurement noise $R$, with $R_{Z}=10^{-27}$.

Figure 8: Effects of mismodelling on the IEM realization

influenced by the mismodelling. Conversely, if the noise is overestimated $\left(R \gg R_{Z}\right)$, the IEM shows an increase in the steering bump, especially if $R_{Z}$ is higher.

\section{Conclusion And Outlook}

The algorithm for simulating the realization of the IEM of a mixed clock ensemble is described in this work. It consists of a very flexible simulation environment, where ensembling scenarios can be tested in a fast way. In particular, the user can form an arbitrary ensemble by choosing the desired number and type of clocks. The simulation uses three different models, reflecting the uncertainty of the modelling in an hardware setup. Two different control strategies are available, namely pole placement and linearquadratic regulator, whose parameters can be freely set. The algorithm simulates each block and step present in a hardware realization.

The results of simulating a homogeneous ensemble have been compared to the corresponding hardware measurement: the two realizations show a good agreement, thus providing a initial validation of the simulation algorithm. A further validation involving a mixed ensemble has to be carried out as soon as the hardware for this scenario is available and running.

Various ensembling scenarios have been simulated involving different settings. This allows to study the effects of each parameter on the resulting realization. Firstly, the ensemble composition is varied. In particular, the generation of Kepler System Time is simulated with an ensemble of 37 clocks. Secondly, different regulators have been applied and their steering parameters changed. Finally, some mismodelling has been introduced either regarding the clock models or the measurement noise. The resulting effects on the realization are shown.

By analyzing the realizations of the simulated scenarios some aspects can be concluded. To achieve a good stability, clocks showing complementary properties have to be used in an ensemble, and the one with the best short term stability should be steered to the corresponding IEM. The control strategy and its parameters $\left(\lambda, W_{r}\right.$ and $\left.\boldsymbol{W}_{Q}, T_{u}\right)$ have to be chosen case by case depending on the properties of the clocks and on the desired results. The mismodelling analysis showed that overestimating the clocks' stability and the measurement noise can lead to increased drift or bumps in the realization. The IEM generation seems particularly sensible to the drift (Fig. 8a). It could be therefore reasonable to have clock models with better stabilities than the respective measured OADEVs.

This work represents one step towards the hardware realization of a mixed clock ensemble. Future investigations cover therefore this objective and complete the previous works. In particular, new steering techniques and new parameters could be used to improve the steering performance and decrease the negative effects of the steering process. Then, as soon as the hardware for a mixed ensemble 
is available, the simulation can be completely validated. Subsequently, the robustness of the composition must be assessed both in simulation and in hardware. This leads to the development and implementation of active techniques of fault detection and identification, which can make the system more robust against failures and unpredictable behaviors.

\section{ACKNOWLEDGMENT}

This work is supported by the Helmholtz-Gemeinschaft Deutscher Forschungszentren e.V. under grant number ZT0007 (ADVANTAGE, Advanced Technologies for Navigation and Geodesy).

\section{REFERENCES}

[1] K. R. Brown Jr., "The theory of the GPS composite clock," in Proceedings of the 4th International Technical Meeting of the Satellite Division of The Institute of Navigation (ION GPS), pp. 223-242, ION, ION, 1991. Albuquerque, NM, September 1990.

[2] M. Gödel and J. Furthner, "Robust ensemble time onboard a satellite," in Proceedings of the 48th Annual Precise Time and Time Interval Systems and Applications Meeting, pp. 26-43, ION, ION, Jan. 2017. 30. Jan. - 1. Feb. 2017, Monterey, US.

[3] M. Gödel, T. Schmidt, and J. Furthner, "Kalman filter approaches for a mixed clock ensemble," in Proceedings of the 2017 European Frequency and Time Forum and International Frequency Control Symposium (EFTF-IFCS 2017), IEEE, IEEE, July 2017. July 10th July 13th 2017, Besançon, France.

[4] C. Trainotti, T. D. Schmidt, and J. Furthner, "Comparison of clock models in view of clock composition, clock steering and measurement fitting," in Proceedings of the Annual Precise Time and Time Interval Meeting (PTTI), (Reston, Virginia), pp. 265-283, Jan. 2019.

[5] J. Davis, C. Greenhall, and P. Stacey, "A kalman filter clock algorithm for use in the presence of flicker frequency modulation noise," Metrologia, vol. 42, no. 1, pp. 1-10, 2005.

[6] T. D. Schmidt, M. Gödel, and J. Furthner, "Investigation of pole placement technique for clock steering," Proceedings of the 49th Annual Precise Time and Time Interval Systems and Applications Meeting, pp. 22-29, 2018.

[7] T. D. Schmidt, C. Trainotti, and J. Furthner, "Comparing clock steering technique performances in simulations and measurements," in Proceedings of the 50th Annual Precise Time and Time Interval Systems and Applications Meeting, (Reston, Virginia), pp. 290-298., Jan. 2019.

[8] M. Gödel, T. D. Schmidt, and J. Furthner, "Comparison between simulation and hardware realization for different clock steering techniques," Metrologia, 2019.

[9] P. Koppang, D. Johns, and J. Skinner, "Application of control theory in the formation of a timescale," tech. rep., NAVAL OBSERVATORY WASHINGTON DC, 2004.

[10] P. A. Koppang, "State space control of frequency standards," Metrologia, vol. 53, no. 3, p. R60, 2016.

[11] L. A. Breakiron and P. Koppang, "Frequency steering of hydrogen masers," in Frequency Control Symposium, 1996. 50th., Proceedings of the 1996 IEEE International., pp. 1113-1122, IEEE, 1996.

[12] P. Koppang and R. Leland, "Linear quadratic stochastic control of atomic hydrogen masers," IEEE transactions on ultrasonics, ferroelectrics, and frequency control, vol. 46, no. 3, pp. 517-522, 1999.

[13] S. T. Hutsell and P. Koppang, "USNO alternate master clock steering," in 32nd Annual Precise Time and Time Interval (PTTI) Meeting, (Reston, VA), pp. 289-300, Nov. 2000.

[14] P. Koppang, D. Matsakis, and M. Miranian, "Alternate algorithms for steering to make GPS time," in Proceedings of ION GPS, pp. 933-936, 2000.
[15] D. W. Allan, "Time and frequency (time-domain) characterization, estimation, and prediction of precision clocks and oscillators," in IEEE Transactions on Ultrasonics, Ferroelectrics, and Frequency Control, vol. 34, pp. 647-654, Nov. 1987.

[16] C. A. Greenhall, "A review of reduced kalman filters for clock ensembles," IEEE transactions on ultrasonics, ferroelectrics, and frequency control, vol. 59, no. 3, 2012.

[17] C. Zucca and P. Tavella, "The clock model and its relationship with the allan and related variances," IEEE Transactions on Ultrasonics, Ferroelectrics, and Frequency Control, vol. 52, pp. 289-296, Feb. 2005.

[18] C. Günther, "Kepler - A Concept for a Future System for Navigation, Geodesy and Metrology." Journal of Navigation (submitted).

[19] C. Günther, "Kepler - Satellite Navigation without Clocks and Ground Infrastructure," in Proceedings of the 31st International Technical Meeting of The Satellite Division of the Institute of Navigation (ION GNSS+ 2018), pp. 849-856, 092018.

[20] T. D. Schmidt, C. Trainotti, J. Isoard, G. Giorgi, J. Furthner, and C. Günther, "Composite Clock Algorithms for System Time in Global Navigation Satellite Systems," in Proceedings of the 31st International Technical Meeting of the Satellite Division of The Institute of Navigation (ION GNSS+ 2018), (Miami, Florida), pp. 963-967, Sept. 2018. 